tion. A response to nanogram quantities of organophosphate pesticide had been obtained by increasing the electrode temperature but this had resulted in rapid loss of selectivity and base-line stability; similar characteristics were found when other alkali metal salts were used and when the salt was introduced into the flame at various positions. Some reduction in the rapid loss of selectivity at high-sensitivity settings had been obtained by using a tube of thin platinum $1 \mathrm{~mm}$ in diameter filled with sodium sulphate as the 'pick-up' electrode. There was considerable scope for development of this detector and the leak detector for use as selective detectors in gas chromatography.

Using the chromatograms obtained for a mixture of $n$-alkane, iso-alkane and alicyclic hydrocarbons, Dr. D. R. Deans (Imperial Chemical Industries, Ltd., Heavy Organic Chemicals Division, Billingham), in the third paper presonted, showed that superior separation of certain compononts difficult to separate could be obtained by using two columns of different type in series and backflushing part of the sample through the first column at some eritical stage during the elution. The degree of separation which could be achieved was better than could be obtained by straightforward elution through either of the columns used alone or in series. In addition to this novel applica. tion of backflushing there was also its more conventional use in conjunction with pre-columns for isolating heavy components. Despite these applications, the technique was not widely used and Dr. Deans thought this was due to the mechanical difficulties of operating taps enclosed in ovens and the attendent contamination of sample with grease and increase in dead volume causing band spreading. These objections had been overcome by using a system in which a capillary $T$-junction was used to connect the two series columns with one $\lim b$ of the junction extending outside the oven to the control system. Similarly the controls for injection and backflush vent were outside the oven (for details see $J$. Chromatog., 18, 477; 1965).

The combinations of columns (two packed, two capillary or packed and capillary in series), of liquid-phase type on the columns, of cutting and of treating the backflush (to vent or back to the column system) were innumerable. Fractions or compounds monitored out of the stream at some intermediate stage could be subjected to in-line reaction using micro-reactors or pyrolysis units. A fascinating example of this type of application was described in which the identification of the components of a complex mixture of heptenes, for which pure compounds for use as references were not available, was achieved by hydrogenating monitored peaks and re-chromatographing them against the more readily available saturated hydrocarbon reference compounds. Operation of the various systems described was considered to be very simple in practice and a switching unit had been constructed for connexion to any existing chromatograph allowing all possible schemes of operation to be used.

Mr. M. B. Evans (Natural Rubber Producers' Research Association) described his work concerned with the role of the solid support in retention measurement. One aspect which had been investigated was the apparent increase in polarity of non-polar liquid phases when used under conditions where oxygen is not rigorously excluded from the column. The retention characteristics of $\mathrm{a}$ wide range of solute types on freshly prepared squalane columns to which known weights of high molecular weight alcohols, aldehydes and ketones had been added were compared with retentions on a squalane column which had been deliberately oxidized at $100^{\circ} \mathrm{C}$; for the columns with added oxygenated compounds there was evidence to show that retention indices are linearly dependent on oxygen content, whereas for oxidized columns there was an initial drop in retention for polar solutes (also a pronounced reduction in peak tailing) followed by an increase. It was suggested that the initial drop observed was due to the first formed strongly polar compounds deactivating the surface and the values at the minimum of the curve corresponded to the retention index due to true partitioning in the squalane.

Evaluation of a number of glycols as support deactivators led to the conclusion that diglycerol was most efficient and its use had enabled improvements in peak symmetry and reduced solute isomerization and decomposition. Detailed investigation of the effect of diglycerol-treated support on the retention of wealkly polar solutes by dinonyl phthalate and squalane phases showed retention to be almost independent of the ratio of liquid phase to the diglycerol deactivator; the retention of polar solutes such as alcohols was found to display marked dependence similar to that observed when squalane was oxidized.

It was considered feasible that the extent of the initial high retention of polar solutes when low liquid phase loadings were used could be a measure of the support activity. The evaluation of a wide range of support material using squalane as the liquid phase and a wide range of solutes at constant sample size provided convincing evidence that support activity could be measured in this manner and indicated that a commercially available silane-treated product was the least adsorptive of those investigated. It was also considered that an evaluation of the support could be made by the reversed procedure of assessing the extent of the deactivation of polar groups of the liquid phase by the support at constant proportion of liquid phase, thus the highest retention index for a polar solute should be given by the least active support. This was confirmed experimentally using polyethyiene glycol liquid phase on the same range of supports as were used for the other investigation.

The final paper of the symposium was presented by Dr. J. F. K. Huber (University of Amsterdam) under the title "Two-Dimensional Gas Chromatography". He discussed factors relevant to the identification of components using two columns having different liquid phases as coatings. After listing the various forms of retention by which a solute could be characterized, Dr. Huber examined in some detail the factors affecting the accuracy of such data, among which were effects due to interfaces, pressure, flow changes and non-linearity of the partition isotherm. The results obtained by sample injection into two different types of column could be correlated by com. bining relative peak area values and known retention values although one source of error was the presence of masked peaks. With two columns combined so that fractions of the effluent from the first column were injected into the second column more definite results could be obtained as well as enrichment of a trace relative to a main component.

C. G. Scot'T

\title{
URBAN TRAFFIC PROBLEMS
}

\begin{abstract}
A MEMORANDUM issued by the Ministry of Transport/Scottish Development Department deals with the principles of applying traffic engineering techniques to urban road traffic problems*. It is designed to enable

* Ministry of Transport/Scottish Development Department. Advisory Memorandum on Orban Traffic Engineering Techniques. Pp. xi + 92. (London:
\end{abstract} H.M.S.O., 1965.) 78.6d. net. suitable short- to medium-term solutions to be found for urgent traffic problems, while providing a useful guide to the wide range of traffic engineering methods and techniques, many of which must be used in formulating the long-term traffic solutions which will be incorporated in comprehensive land use/transport plans. It does not attempt to give guidance on the surveys needed for 
preparing these plans. The shorter-term traffic solutions should not be looked at in or by themselves, howevor, but should be viewed so far as possible in relation to parking policies, to the needs of environmental as well $u$ traffio management and to tho likely ultimate pattern of town roads. Together they constitute the broad approach to the problem of traflic in towns; in particular, any short-term proposals which would determine road widths or alignments for a long time ahedid should be carefully considered in this wider context.

The memorandum includes a report on a preliminary reconnaisance to identify the nature of traffic problems, the information available on traffic data, accidents and land-uses, and the extent to which further information is needed. This is followed by a section on mothods of taking traffic censuses, origin and destination surveys, surveys of public transport, measurements of speed, parking surveys and of collecting accident data. In the third section, the processing and presentation of survey information ready for some formulating scheme are considered, and in the fourth, the design of short-, mediumor long-term schemes to meet the needs of both traffic and road safety. The fifth section considers methods for comparing the advantages and disadvantages of alterna. tive proposals, including the economic assessment of road improvements, and, in the sixth soction, investigations needed to vorify the adequacy of the measures taken and to reveal any need for further measures aro discussed. Finally, the value of quantitative assessment of traffic problems and improvement schemes, the need to study and train engineers in the methods of meeting traffic requirements and to take advantage of now, or little-used, techniques are considered.
New methods of meeting traffic problems, such as new types of signal-controlled pedostrian crossings, "give way to traffic from the right" signs at roundabouts, the use of vehicle presence detectors, tidal traffic working and area traffie control, are likely to come increasingly into use as their possibilities are explored, with also increasing application of pedestrian segregation, channelization, urban motorways, and grade-separated systems. Shortterm measures may offor much relief of congestion at little cost, and waiting restrictions, banning of right turns, or one-way systems often increase road capacity by 20 per cent or more. Improvemonts of 20 to 50 per cent can often be effected at signal-controlled junctions, by rephasing and simplifying the traffic pattern, by restricting certain movements, or by small carriageway widenings at the signal approaches, as well as through increasing use of the more flexible modern traffic signal controllers to meet peak-hour demands.

With medium-torm measures, the emphasis should be on junction improvements, provision of roundabouts, new signal-controlled junctions and widening roads, particularly at junctions or bottlenecks. In narrow streets lay-bys for buses or delivery vehicles can be of great value in promoting a freer flow. 'I'raffic engineering techniques can help longer-term moasuros which require programming in order of priority. In determining priorities and in designing various schemes for new or improved roads and junctions and general road layout, to meet the future requiromonts of a town, it is emphasized that in establishing environmental areas and freeing them of unnecessary traffic, the traffic on the primary road network will bes increased, and the network must therefore be designed to take heavier flows.

\section{SOCIAL INFLUENCES ON MEASURED INTELLIGENCE}

T HE Novernber issuc of Educational Research (8, No. 1) contains fivo separate papers on various aspects of the influence of social factors on the measured intelligence quotiont (I.Q.). Taken together, they are of outstanding public interest. One paper is entitled "Further Evidence on the Effect of Date of Birth on Subsequent School Performance", by R. Freyman, and is based on a study of: (a) 3,419 children born during 1952-55 from eight streamed junior schools in different parts of Middlesbrough; (b) 4,120 children born during 1953-55 from seven streamed secondary modern schools, four grammar schools and two other selective secondary schools; (c) 364 junior school children attending remedial reading groups; (d) 312 children reforred to the child guidance service; and (e) 156 children attending the day spocial school for educationally subnormal (E.S.N.) children. Among the children in streamed junior schools, of those born during the period Septomber to Decomber, 39.7 por cent were in $A$ classes, 25.8 per cent in $B$, and 22.4 per cent in $C$, while of those born in the period May to August, 28.2 per cent wore in $A, 36 \cdot 6$ por cent in $B$, and $43 \cdot 7$ per cent in $C$ classes. These differences were statistically highly significant, along with the finding that the percentage in the $A$ stream increased with length of schooling.

Similar results were obtained from all the children attending secondary schools. of those born during September to December, 36 per cent were in selective schools and 28.4 per cent in secondary modern schools, whereas, of the ones born during May to August, 32.3 per cent were in solcctive schools and 36.1 in secondary modern schools. Again, a larger proportion of younger than of older children obtained low and average marks.

of the children in remedial reading groups, 46.7 were born during May to August, and 27.8 per cent during January and April periods, a discrepancy which was wider still among those referred to the educational psychologist because of backwardnoss in reading. The children attending the local E.S.N. school produced less clearly cut differences.

From his survey, Freyman concludes that the date of birth exerts a significant influence on a child's school grading, and that at junior level, streaming in schools tends to perpetuate differences due to date of birth. The disadvantages of the children born during May to August wero greatest in those referred because of difficulties in reading; and "secondary psychological symptoms such as lack of confidence and emotional maladjustment were often displayed".

It might be thought that these discrepancies arise because children borm in September to December are almost 8 years old when transferred from infant to junior schools as compared with the 7 or 7 and 4 months age of thoso born in the summer. But in itself, that factor, although real, can be allowed for; it does not account for the whole of the difference, which seerns to be determ ined by some specific condition, not simply delayed mental development; and Freyman calls for a new educational deal for children born during the summer, which might include longer time in the infant school and more flexible forms of educational organization.

In his paper on "Date of Birth and Scholastic Pcrformance", D. A. Pidgeon is concernod with the same kind of theme whilo carrying the discussion somewhat further. He makes the point that children who have hac more schooling will have lenrned more and in examinations are likely to give better results; but in addition, children born in summor usually rernain the youngest in their group throughout the junior school, a situation which imposes additional stress. Supporting evidence comes from Sweden.

It is a moot point whether, as a whole, the innate intelligence varies as between childran born at different 